\title{
Adenoma pleomorfo del septum nasal, dos casos clínicos y revisión del tema
}

\author{
Pleomorphic adenoma of the nasal septum. Two clinical cases
}

Javiera Mercado $\mathrm{A}^{\mathbf{1}}$, Víctor Mercado $\mathbf{M}^{2}$, Ignacio Fernández $\mathrm{Z}^{3}$, Humberto Vallejos $\mathrm{A}^{\mathbf{4}}$.

\begin{abstract}
RESUMEN
El tumor benigno más frecuente de las glándulas salivales mayores es el adenoma pleomorfo. Además de las glándulas salivales mayores, también puede ocurrir en las glándulas salivales del paladar duro y blando. Raros casos se han reportado en la nasofaringe, orofaringe, hipofaringe y laringe. Adenomas pleomorfos intranasales son muy infrecuentes y pueden ser mal diagnosticados debido a que tienen mayor celularidad mioepitelial y poco estroma mixoide, en comparación con los que se localizan en otros lugares. Presentamos dos raros casos de adenomas pleomorfos del septum nasal y analizamos los hallazgos patológicos y el manejo clínico. Se revisa la literatura en relación al tema.
\end{abstract}

Palabras clave: Adenoma pleomorfo, septum nasal, glándula salival.

\begin{abstract}
The most common benign tumor of major salivary glands is the pleomorphic adenoma. In addition to the major salivary glands may also occur in the salivary glands of hard and soft palate. Rare cases have been reported in the nasopharynx, oropharynx, hypopharynx and larynx. Intranasal pleomorphic adenomas are very rare and can be misdiagnosed because they have greater myoepithelial cellularity and little myxoid stroma, compared with those located elsewhere. We report two rare cases of pleomorphic adenomas of the nasal septum and analyze the pathological findings and clinical management. We review the literature on the subject.
\end{abstract}

Key word: Pleomorphic adenoma, Nasal septum, Salivary gland.

\footnotetext{
'Interna de Medicina, Facultad de Medicina, Universidad de Valparaíso.

2Medico Otorrinolaringólogo, Clínica Reñaca, Viña del Mar.

${ }^{3}$ Estudiante de Medicina, Escuela de Medicina, Universidad Andrés Bello, Viña del Mar.

${ }^{4}$ Medico Anatomopatólogo, Hospital Carlos Van Buren, Valparaíso.
} 


\section{INTRODUCCIÓN}

Los tumores de las glándulas salivales son infrecuentes, sólo representan $3 \%$ del total de los tumores. De éstos, el $85 \%$ a $90 \%$ se encuentra en las glándulas salivales mayores y $10 \%$ a $15 \%$ en las menores ${ }^{1,2}$.

El adenoma pleomorfo (AP) es el tumor benigno más frecuente de estas glándulas, correspondiendo a $65 \%{ }^{2}$, siendo más frecuente en la parótida $60 \%-70 \%$, glándulas submaxilares $40 \%$ a $60 \%$ y glándulas salivales menores de la cavidad oral $40 \%$ a $70 \%$. En cambio, es rara su localización en las glándulas sublinguales, labios, paladar duro y blando, glándulas lagrimales y en el canal auditivo externo ${ }^{3-7}$. En relación a la fosa nasal, es muy infrecuente encontrarlo, el primer reporte de un caso en la literatura fue el año 1929, por Denker y Kahler $^{8}$. Luego se publican 40 casos por Spiro ${ }^{9}, 40$ por Campagno y Wong ${ }^{3}, 41$ por Suzuki ${ }^{6}$ y presentaciones de casos aislados. Reportamos dos casos clínicos y realizamos una breve revisión de la literatura.

\section{CASO CLÍNICO 1}

Paciente sexo femenino de 53 años de edad, consultó por una "desviación" y obstrucción nasal izquierda, de aproximadamente 7 meses de evolución. A la inspección, se apreció una deformación

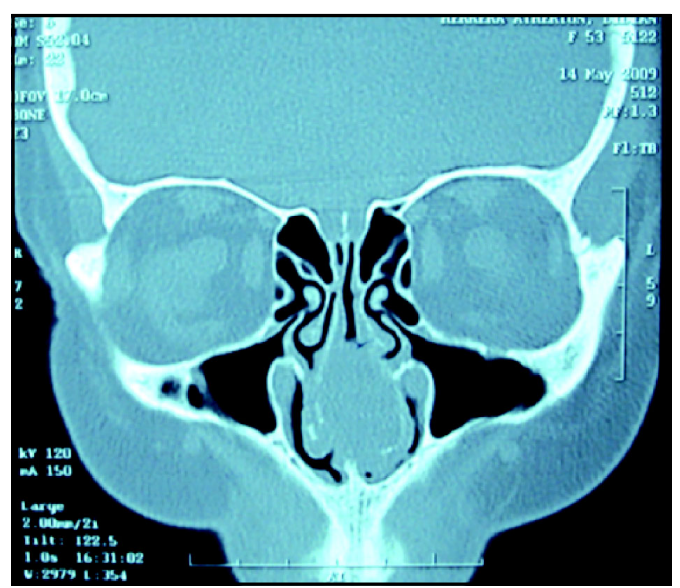

Figura 1. de la pirámide nasal y a la rinoscopía anterior en la fosa nasal izquierda se visualizó una masa de aspecto tumoral de color rojo pálido de $3 \mathrm{~cm}$ de diámetro mayor que ocupaba el tercio medio de la fosa nasal en relación al septum osteocartilaginoso.

La tomografía computarizada de cavidades paranasales informó una masa voluminosa redondeada, hipodensa de $30 \mathrm{~mm}$ de diámetro ántero posterior y $23 \mathrm{~mm}$ de diámetro transverso, que ocupaba la fosa nasal izquierda con desviación del septum nasal a derecha (Figuras 1 y 2). Con estos antecedentes se realiza un degloving izquierdo, con un tiempo quirúrgico de 45 minutos donde se reseca el tumor con límites amplios y fácil clivaje, lo que permitió conservar el tejido septal osteocartilaginoso adyacente al tumor, no así el pericondrio del septum que se incluye en la pieza operatoria. La histopatolología del espécimen, mostró la presencia de un estroma fibromixoide con células epiteliales y mioepiteliales, sin formación de glándulas, característico de AP (Figura 3).

Al año de seguimiento, la paciente se encontraba asintomática, con fosa nasal izquierda anatómica y funcionalmente normal.

\section{CASO CLÍNICO 2}

Paciente de sexo femenino de 28 años sin antecedentes mórbidos conocidos consultó por obstruc-

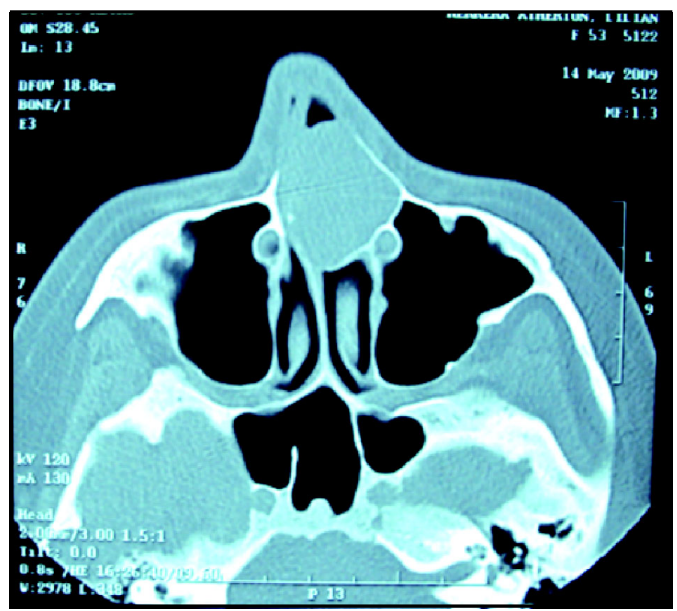

Figura 2. 


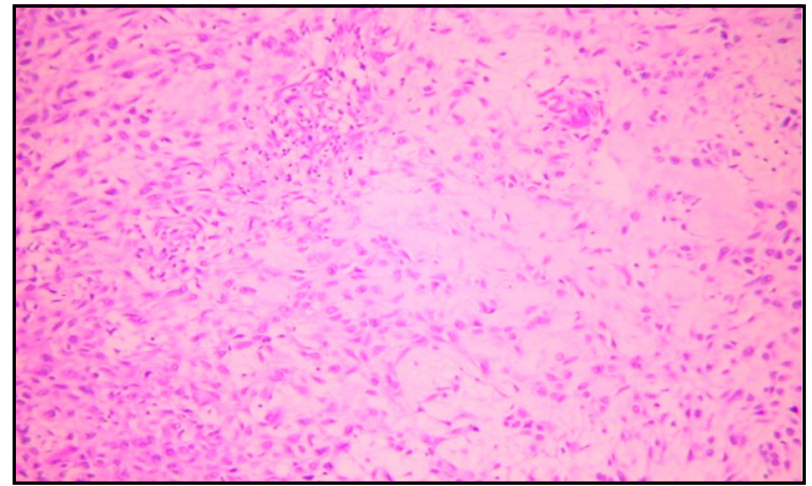

Figura 3. Muestra teñida con hematoxilina \& eosina. Visión panorámica del tumor. El aumento es de 4 x. Se observa estructuras fibromixoide en el estroma, característico de adenoma pleomorfo con células epiteliales y mioepiteliales, sin formación de glándulas.

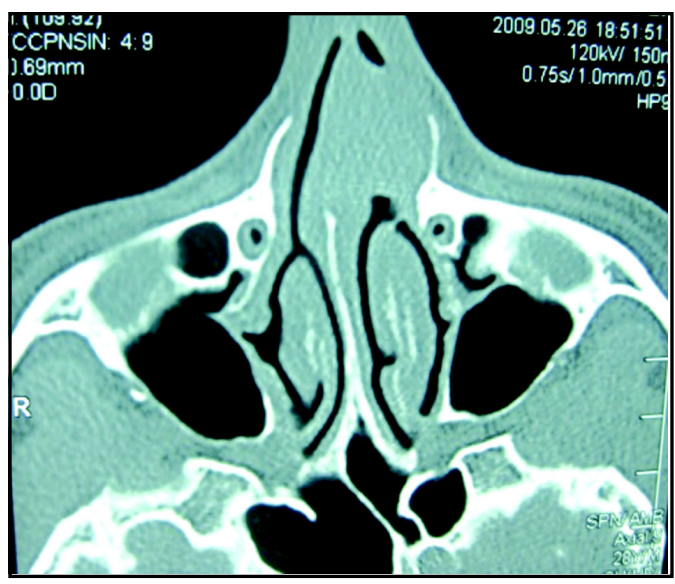

Figura 4.

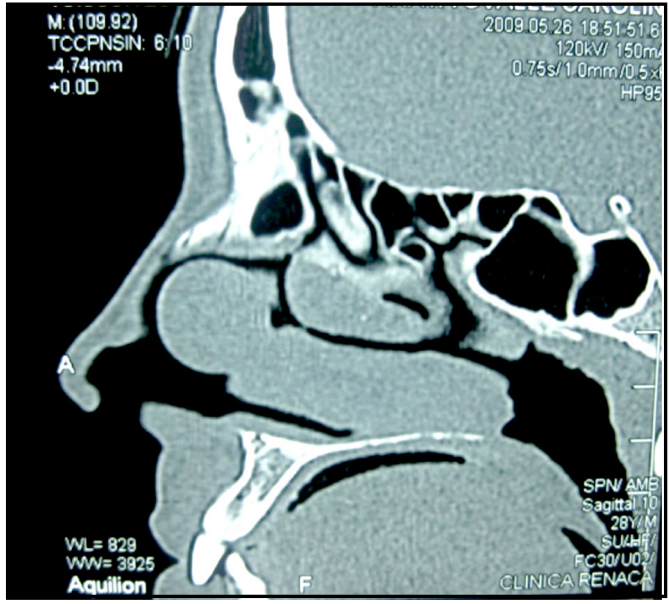

Figura 5.

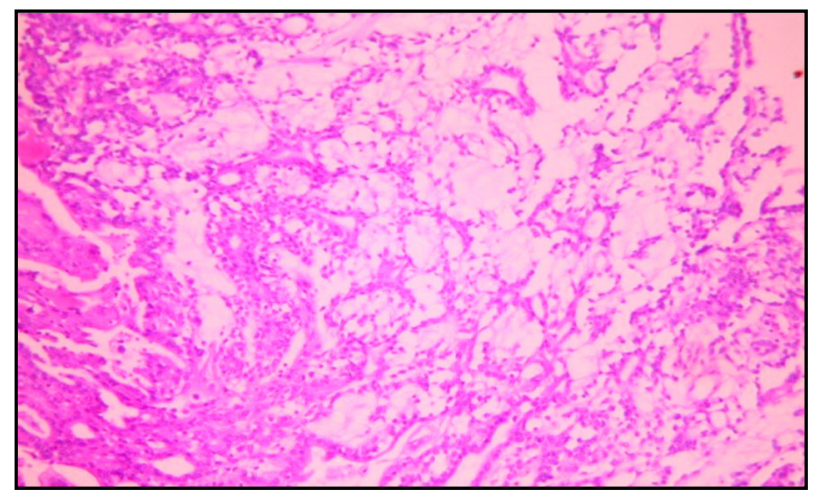

Figura 6. Muestra teñida con hematoxilina \& eosina. El aumento es de $10 x$. Se observan estructuras epiteliales que forman glándulas o túbulos en la esquina inferior izquierda y también áreas extensas de estroma mixoide que separa las células epiteliales y mioepiteliales. 
ción nasal izquierda de 6 meses de evolución. A la inspección se observó una deformación del surco nasogeniano izquierdo y la rinoscopía anterior pesquisó a nivel de la fosa nasal izquierda, una masa redondeada de color rojo pálido de aproximadamente $2 \mathrm{~cm}$ en su diámetro mayor, que ocupaba el tercio anterior de la fosa nasal en relación al septum, desplazando el cornete inferior. La fosa nasal derecha se encontraba estrecha por el desplazamiento que la masa tumoral producía sobre el septum.

La tomografía computarizada de cavidades paranasales, practicada en mayo de 2009, informó un nódulo dependiente de la porción cartilaginosa anterior del tabique nasal, ocupando parte del vestíbulo, probablemente benigno, de aproximadamente $20 \mathrm{~mm}$ de diámetro mayor con escoliosis del tabique nasal, determinando una menor amplitud de fosa nasal derecha (Figuras 4 y 5 ).

En julio de 2009 debió diferirse la cirugía porque la paciente cursaba embarazo de 4 semanas. Nueve meses después, en la evaluación clínica preoperatoria llama la atención un aumento de tamaño de la masa y una mayor deformación del surco nasogeniano. Por medio de la técnica sublabial de Denker se realizó la resección quirúrgica del tumor con límites amplios, en un tiempo quirúrgico de 45 minutos. Se conservó el cartílago septal y la pared medial de la fosa nasal.

La histopatología del espécimen operatorio reveló estructuras epiteliales que forman glándulas 0 túbulos y áreas extensas de estroma mixoide que separa las células epiteliales y mioepiteliales, compatible AP (Figura 6).

A los 6 meses de seguimiento, la paciente no presentó síntomas de obstrucción nasal, con fosa nasal izquierda anatómica y funcionalmente normal.

\section{DISCUSIÓN}

La ubicación más frecuente del AP de la cavidad nasal, es el septum $80 \%{ }^{3,10}$, y el $20 \%$ restante corresponde a la cavidad nasal lateral ${ }^{11}$. La mayoría de estos tumores intranasales ocurren entre la tercera y sexta década de vida ${ }^{3,12}$. Hay una discordancia en la literatura, ya que algunos autores mencionan que son más frecuentes en mujeres ${ }^{6}$, mientras que otros establecen que no hay diferen- cia entre ambos sexos ${ }^{3}$. No ha sido descrita una correlación con exposición ocupacional ni tampoco con compuestos químicos tóxicos inhalados ${ }^{13}$.

El cuadro clínico del AP endonasal es inespecífico. Se presenta como una masa, la mayoría de las veces en relación al septum ${ }^{3}$, unilateral, indolora, que produce una obstrucción crónica, como principal sintomatología. Con menor frecuencia puede presentar epistaxis, deformación nasal externa y sintomatología de una sinusitis crónica ${ }^{5,12}$. Al examen clínico se aprecia una masa polipoidea, lisa, lobulada y con una cápsula bien definida ${ }^{12}$, lo que puede provocar que sean confundidos muchas veces con pólipos nasales. El tamaño AP varía desde $0,7 \mathrm{a} 7 \mathrm{~cm}^{13}$.

Como en todo estudio de masa endonasal, es necesaria la imagenología, ya sea tomografía computarizada (TC) y/o resonancia magnética (RM), mas, la confirmación diagnóstica definitiva será siempre histopatológica. En la TC de cavidades paranasales, se visualiza generalmente una masa lobulada, lisa, emergiendo ya sea del septum nasal 0 de la pared lateral de la cavidad, con un patrón generalmente expansivo lo que sugiere una lesión benigna ${ }^{6}$. Con contraste se visualiza una masa heterogénea, y en algunas oportunidades se pueden encontrar calcificaciones. La lesión en ocasiones se extiende a los senos paranasales erosionando sus paredes. En la RM, Ios AP pequeños de glándulas salivales mayores se ven como una masa homogénea de baja intensidad en T1 y alta en T2. Los tumores de mayor tamaño en cambio, tienden a desarrollar un contorno lobular y ocasionalmente tienen una calcificación distrófica u osificación. No son homogéneos, con una intensidad baja a intermedia en $\mathrm{T} 1$ y de intermedia a intensa en T2. También puede haber zonas de necrosis que tienen baja intensidad en $\mathrm{T} 1 \mathrm{y}$ alta en $\mathrm{T} 2^{14}$.

Embriológicamente las glándulas salivales derivan de abultamientos del epitelio bucofaríngeo en el embrión. Los filamentos del epitelio glandular invaden el tejido conectivo de la cápsula, siendo esto un posible responsable de la formación del tejido ectópico salival en distintos sitios ${ }^{15}$. En cuanto a su etiopatogenia, existen tres potenciales orígenes descritos en la literatura. El primero propone un origen de restos embrionarios del órgano vomeronasal (órgano de Jacobson) ${ }^{16}$. Otro posible 
origen es de las células epiteliales ectodérmicas embrionarias aberrantes ${ }^{17}$, y por último el tejido glandular salival que puede sufrir una transformación neoplásica que es inducida por el virus del papiloma humano ${ }^{18}$. También existen estudios publicados que podrían explicar la participación del virus de Epstein Barr en el desarrollo de estos tumores, en esta particular localización ${ }^{19}$.

La histología del AP intranasal es similar al de otras localizaciones, teniendo mayor celularidad mioepitelial. Tiene un componente mesenquimático y epitelial, este último tiene un patrón trabecular, con un estroma de menor tamaño que puede ser clasificado como mixoide, condroide, fibroide u osteoide. Los tumores con patrón mixoide son los que más se asocian a tumores recurrentes, ya que puede ser sembrado en el campo quirúrgico, formando un nido para la recurrencia ${ }^{20}$. En la literatura existe un caso descrito con una diferenciación de músculo esquelético ${ }^{21}$ y otro caso con neoformación de osteoide que simulaba un osteosarcoma ${ }^{22}$.

El riesgo potencial de malignizarse es de alrededor del 6\%, y es más frecuente que suceda en el sexo femenino ${ }^{23}$. El riesgo se ve aumentando con el retraso del diagnóstico. Se ha reportado un caso de una metástasis cervical de un AP recurrente de la cavidad nasal histológicamente benigna, luego de 17 años de haberlo extirpado. Se cree que durante la escisión de la masa nasal se sembraron células tumorales ${ }^{24}$. Dos neoplasias del septum nasal han sido descritas como carcinomas ex-AP de la mucosa nasal. En ningún caso hubo metásta$\mathrm{sis}^{25}$. Un tercer caso descrito fue un carcinoma exAP con diferenciación adenoidequístico y carcinoescamoso. Luego de la cirugía y radioterapia, el paciente de igual manera falleció por metástasis cerebral, hepática y ósea ${ }^{26}$.

Los diagnósticos diferenciales pueden ser bastantes y muy variados, incluyendo desde tumores benignos hasta malignos, por lo que es muy importante hacer un diagnóstico certero y a tiempo (Tabla 1).

En relación al tratamiento de los AP del septum nasal, la intervención de elección es la extirpación quirúrgica con márgenes libres, teniendo un bajo índice de recidivas de $10 \%{ }^{3}$. Las técnicas más aceptadas por ser rápidas y de baja morbilidad son
Tabla 1. Diagnósticos diferenciales ${ }^{10,21}$

Lesiones congénitas en niños
Quistes dermoides
Encefaloceles externos
Gliomas nasales
Adolescentes
Angiofibroma juvenil
Adultos
Papiloma Invertido
Plasmocitoma
Cordoma
Oncocitoma
Fibrohistiocitoma
Neuroblastomas olfatorios
Tumores neuroendocrinos
Hemangiopericitoma
Rabdomiosarcoma
Mioepiteliomas
Adenocarcinomas polimorfos de bajo grado
Carcinoma adenoide quístico
Pólipos nasales
Otros

la maxilotomía medial de Denker, Degloving medio facial y la endoscópica ${ }^{27}$, siendo esta última la que ofrece menos morbilidad en manos expertas. Los beneficios de la técnica de Denker es que por medio de esta vía, se tiene una amplia exposición de los límites de la lesión. En los casos expuestos se eligieron las técnicas de Degloving y Denker, debido al claro conocimiento de esta técnica, su corto tiempo quirúrgico y la mínima morbilidad, sumado al hecho del desconocimiento del diagnóstico histopatológico del tumor y su inusual ubicación.

\section{CONCLUSIONES}

El AP de cavidad nasal es poco frecuente, y se diferencia de aquellos de localización parotídea, por su mayor celularidad, tener menor porcentaje de recidivas y ser menos agresivos. Debe siempre tenerse en cuenta como diagnóstico diferencial en una masa obstructiva de la fosa nasal, que ocupa principalmente el septum nasal. 


\section{BIBLIOGRAFÍA}

1. Som PM. Sinonasal cavity. In: Som PM, Bergeron RT (ed) Head and neck imaging. $2^{\text {nd }}$ edn. Mosby-Year Book, St Louis, 1991; 51-276.

2. Prager da, Weiss mH, Buchalter WL, et al. Pleomorphic adenoma of the nasal cavity. Ann Otol Rhinol Laryngol 1991; 100 (7): 600.

3. Campagno J Wong RT. Intranasal mixed Tumor (pleomorphic adenoma). A clinicoptahologic study of 40 cases. Am J Clin Pathol 1977; 68: 213-8.

4. Trotoux L, Lefebre B. Pathologie des glandes salivaires. Enc Med Chir OrI Paris: Masson; 1979.

5. Campos a, Bagan JV, Zapater E, Martorell M, BASTERRA J. Adenoma pleomorfo de glándula salivar accessoria. Acta Otorrinolaringol Esp 1995; 46: 331-4.

6. Suzukı K, Moribe K, Baba S. A rare case of pleomorphic adenoma of lateral wall of nasal cavity, with special reference of statistical observation of pleomorphic adenoma of nasal cavity in Japan. Nippon Jibiinkoka Gakkai Kaiho 1990; 93: 740-5.

7. Lahoz MT, Valerio J, Royo J, Yus C, Camora F. Tumor mixto de conducto auditivo externo. Acta Otorrinolaringol Esp 1990; 41,1: 53-6.

8. Denker A, Kahler 0. Handush der Hals. Nasen ohrenheilkunde 1929; 5: 202.

9. Spiro RH, Koss LG, Hajdu SI, et al. Tumors of minor salivary origin. A clinicopathologic study of 492 cases. Cancer 1973; 31: 117-29.

10. Wenig BL, Scuibba JJ, Cohen A, et al. Pleomorphic adenoma of the nasal septum. Otolaryngol Head Neck Surg 1985; 93: 432-6.

11. Sánchez-Alcón MD, Herrero E, Iñesta J, Rodrígo 0, Morera C. Adenoma pleomorfo rinosinusal. Acta Otorrinolaring Esp 1996; 47: 71-3.

12. Clark M, Fatterpekar GM, Mukherul SK, Buenting J. CT of intranasal pleomorphic adenoma. Neuroradiology 1999; 41: 591-3.

13. Sciandra D, F. Dispenza F, Porcasi R, Kulamarva G, SARANItI C. Pleomorphic adenoma of the lateral nasal wall: case report. ACTA Otorhinolaryngologica italica 2008; 28: 150-3.

14. Som PM, Brandwein M. Salivary glands. In: Som
PM, Curtin HD, eds. Head and Neck Imaging $3^{\text {rd }}$ ed. St. Louis, Mo: Mosby; 1996; 879-82.

15. GILL BS, REKHI BM. Pleomorphic adenoma of the nose-A case report. Indian Journal of Otolaryngology 1968; vol XX, No.3: 132-4.

16. SteVenson HN. Mixed tumor of the nasal septum. Ann Otol Rhinol Laryngol 1932; 41: 563-70.

17. Matthew S, Ersner MD, Saltzman M. A mixed tumor of the nasal septum. Report of a case. Laryngoscope 1944; 54: 287-96.

18. Fraile Rodrigo JJ, Martínez Berganza y Asensio R, Yus Gotor C, García Ortín M, García Ortín J. Adenoma pleomorfo de tabique nasal. Revisión. Acta Otorrinolaring Esp 1993; 44: 239-41.

19. Malinvaud D, Couloigner V, Badoual C, Halimi P, BonflLS P. Plemorphic adenoma of de nasal septum and its relationship with Epstein- Barr virus. Auris Nasus Larynx 2006; 33: 417-21.

20. Krolls SO, Boyers RC. Mixed tumours of salivary glands. Long-term follow-up. Cancer 1972; 30: 276-81.

21. Lam PW, Chan JK, Sin VC. Nasal pleomorphic adenoma with skeletal muscle differentiation: potential misdiagnosis as rhabdomyosarcoma. Hum Pathol 1997; 28: 1299-302.

22. Lee KC, Chan JK, Chong YW. Ossifying pleomorphic adenoma of the maxillary antrum. J Laryngol Otol 1992; 106: 50-2.

23. ShAHEEN $\mathrm{OH}$. Benign salivary glands tumors. Scott Brown's Otolaryngol 1997; 5: 1-18.

24. Freeman SB, Kennedy KS, Parker GS, Tatum SA. Metastasizing pleomorphic adenoma of the nasal septum. Arch Otolaryngol Head Neck Surg 1990; 116: 1331-3.

25. Cho KJ, el-Naggar AK, Mahanupab P, Luna Ma, BATSAKIS JG. Carcinoma ex-pleomorphic adenoma of the nasal cavity: A report of two cases. J Laryngol Otol 1995; 109: 677-9.

26. Freeman SR, Sloan P, de Carpentier J. Carcinoma expleomorphic adenoma of the nasal septum with adenoid cystic and squamous carcinomatous differentiation. Rhinology 2003; 41: 118-21.

27. Gana P, Masterson L. Pleomorphic adenoma of the nasal septum: a case report. Journal of Medical Case Reports 2008; 2: 349.

Dirección: Dr. Víctor Mercado Martínez

Avenida Libertad $1348,6^{\circ}$ piso, Viña del Mar

E mail: victormercadom@hotmail.com 